\title{
The impact of maternal gestational weight gain on cardiometabolic risk factors in children
}

\author{
Claudia H. T. Tam ${ }^{1} \cdot$ Ronald C. W. Ma ${ }^{1,2,3} \cdot$ Lai Yuk Yuen $^{4} \cdot$ Risa Ozaki ${ }^{1,2} \cdot$ Albert Martin Li $^{5} \cdot$ Yong Hou ${ }^{1}$ • \\ Michael H. M. Chan ${ }^{6}$. Chung Shun $\mathrm{Ho}^{6}$ - Xilin Yang ${ }^{7}$ Juliana C. N. Chan ${ }^{1,2,3}$ • Wing Hung Tam ${ }^{4}$
}

Received: 5 June 2018 / Accepted: 30 July 2018 / Published online: 17 September 2018

(C) The Author(s) 2018

\begin{abstract}
Aims/hypothesis Accumulating evidence suggests an impact of gestational weight gain (GWG) on pregnancy outcomes; however, data on cardiometabolic risk factors later in life have not been comprehensively studied. This study aimed to evaluate the relationship between GWG and cardiometabolic risk in offspring aged 7 years.

Methods We included a total of 905 mother-child pairs who enrolled in the follow-up visit of the multicentre Hyperglycemia and Adverse Pregnancy Outcome study, at the Hong Kong Centre. Women were classified as having gained weight below, within or exceeding the 2009 Institute of Medicine (IOM) guidelines. A standardised GWG according to pre-pregnancy BMI categories was calculated to explore for any quadratic relationship.

Results Independent of pre-pregnancy BMI, gestational hyperglycaemia and other confounders, women who gained more weight than the IOM recommendations had offspring with a larger body size and increased odds of adiposity, hypertension and insulin resistance (range of $p$ values of all the traits: $4.6 \times 10^{-9}<p<0.0390$ ) than women who were within the recommended range of weight gain during pregnancy. Meanwhile, women who gained less weight than outlined in the recommendations had offspring with increased risks of hypertension and insulin resistance, compared with those who gained weight within the recommended range $\left(7.9 \times 10^{-3}<p<0.0477\right)$. Quadratic relationships for diastolic blood pressure, AUC for insulin, pancreatic beta cell function and insulin sensitivity index were confirmed in the analysis of standardised GWG $\left(1.4 \times 10^{-3}<p_{\text {quadratic }}<\right.$ 0.0282). Further adjustment for current BMI noticeably attenuated the observed associations.

Conclusions/interpretation Both excessive and inadequate GWG have independent and significant impacts on childhood adiposity, hypertension and insulin resistance. Our findings support the notion that adverse intrauterine exposures are associated with persistent cardiometabolic risk in the offspring.
\end{abstract}

Electronic supplementary material The online version of this article (https://doi.org/10.1007/s00125-018-4724-x) contains peer-reviewed but unedited supplementary material, which is available to authorised users.

Wing Hung Tam

tamwh@cuhk.edu.hk

Ronald C. W. Ma

rcwma@cuhk.edu.hk

1 9/F, Lui Che Woo Clinical Science Building, Department of Medicine and Therapeutics, The Chinese University of Hong Kong, Prince of Wales Hospital, 30-32 Ngan Shing Street, Shatin, Hong Kong Special Administrative Region, Hong Kong

2 Hong Kong Institute of Diabetes and Obesity, The Chinese University of Hong Kong, Shatin, Hong Kong Special Administrative Region, Hong Kong

3 Li Ka Shing Institute of Health Sciences, The Chinese University of Hong Kong, Shatin, Hong Kong Special Administrative Region, Hong Kong
$4 \quad$ 1/F, Special Block (Block E), Department of Obstetrics and Gynaecology, The Chinese University of Hong Kong, Prince of Wales Hospital, 30-32 Ngan Shing Street, Shatin, Hong Kong Special Administrative Region, Hong Kong

5 Department of Paediatrics, The Chinese University of Hong Kong, Shatin, Hong Kong Special Administrative Region, Hong Kong

6 Department of Chemical Pathology, The Chinese University of Hong Kong, Shatin, Hong Kong Special Administrative Region, Hong Kong

7 Department of Epidemiology and Biostatistics, School of Public Health, Tianjin Medical University, Tianjin, China 


\section{Research in context}

\section{What is already known about this subject?}

- Malnutrition in utero sustainably shapes the body's structure, function and metabolism and contributes to adult diseases

- Emerging data indicates an increased risk of adverse pregnancy outcomes in women who did not hit the weight gain target of the Institute of Medicine 2009 guidelines

- The role of maternal gestational weight gain (GWG) in offspring's long-term cardiometabolic risk remains unclear

\section{What is the key question?}

- How does maternal GWG affect the offspring's cardiometabolic risk at a young age (7 years)?

\section{What are the new findings?}

- Independent of pre-pregnancy BMI, gestational hyperglycaemia and other confounders, we confirmed that GWG was positively associated with body size and adiposity in offspring

- More importantly, we observed a U-shaped relationship between maternal GWG and increased risks of insulin resistance and hypertension, with higher diastolic blood pressure, greater AUC for insulin and pancreatic beta cell function, and lower Matsuda insulin sensitivity index in children whose mother had gained more or less weight than recommended during pregnancy

- $\quad$ Further adjustment for current BMI attenuated the observed associations

\section{How might this impact on clinical practice in the foreseeable future?}

- Our findings support the idea that an unfavourable gestational environment predisposes offspring to longterm cardiometabolic risk, highlighting the need to optimise pre-conception and pregnancy conditions and develop prevention strategies for children whose mothers gained weight outside of the recommendations during pregnancy

Keywords Adiposity · Cardiometabolic risk factors · Chinese children · Gestational weight gain · Hypertension · Insulin resistance $\cdot$ Maternal pre-pregnancy BMI

\author{
Abbreviations \\ $\mathrm{AUC}_{\mathrm{glu}} \quad \mathrm{AUC}$ for glucose \\ $\mathrm{AUC}_{\text {ins }} \quad \mathrm{AUC}$ for insulin \\ DBP Diastolic blood pressure \\ FPG Fasting plasma glucose \\ FPI Fasting plasma insulin \\ GWG Gestational weight gain \\ HAPO Hyperglycemia and Adverse Pregnancy Outcome \\ IOM Institute of Medicine \\ ISI Insulin sensitivity index \\ IGI Insulinogenic index \\ SBP Systolic blood pressure
}

\section{Introduction}

Type 2 diabetes has been recognised as a familial disease transmitted across generations through genetic, epigenetic and environmental circumstances including in utero exposures. To illustrate the role of early-life exposures in programming of disease, previous studies have reported evidence of an excess maternal inheritance of type 2 diabetes and cardiometabolic risk in offspring compared with the pattern of paternal inheritance $[1,2]$.

Barker's hypothesis, focusing on the association of prenatal undernutrition with the risk of cardiovascular and metabolic disease in adult life, has been corroborated by the study of individuals exposed to the Dutch famine [3]. More recently, the Developmental Origins of Health and Disease (DOHaD) hypothesis extended this concept to maternal overnutrition [4]. A series of elegant studies into the Pima Indian population strongly supported this hypothesis, demonstrating the adverse effects of intrauterine exposure to hyperglycaemia on children's adiposity and glucose metabolism [5]. One possible mechanism involved in these hypotheses is that the fetus's adaptations to malnutrition in utero to maximise its chances for short-term survival may sustainably perturb the regulation of energy-balance and energy-sensing pathways via epigenetic modification, 
leading to the subsequent development of obesity and related metabolic disorders in adulthood, which may then be transmitted into the next generation [6].

With the recent upward shift of maternal weight gain during pregnancy, increasing evidence suggests a separate role for maternal gestational weight gain (GWG) on offspring cardiometabolic risk (e.g. inadequate and excessive GWG are considered to reflect undernutrition and overnutrition, respectively). The majority of studies addressing GWG in metabolic disorders have found a positive association between maternal GWG and adiposity in offspring [7, 8], although some have reported a U-shaped relationship [9, 10]. A few studies have also investigated the effect of GWG on other cardiometabolic risk factors, including fasting plasma glucose (FPG) and insulin (FPI) levels, HOMA-IR, blood pressure and lipid profile in childhood [11-13], adolescence [14] and adulthood [15-17].

To help clinicians monitor an appropriate GWG, the Institute of Medicine (IOM) reviewed the impact of weight gain during pregnancy on both maternal and fetal outcomes, and in 2009 established new guidelines for optimal GWG according to pre-pregnant BMI [18]. The IOM has, however, recognised that more research is required to study the role of maternal GWG in long-term health in the offspring.

By using follow-up data on mother-child pairs from the multicentre Hyperglycemia and Adverse Pregnancy Outcome (HAPO) study, procured at the Hong Kong study centre [19], the current study aimed to examine the relationships between maternal GWG and cardiometabolic risk factors in offspring, measured at 7 years of age, adjusting for a number of potential covariates. Birthweight is often used as an indicator of fetal conditions (e.g. growth and nutrition) in utero. It has been suggested that the relationship between birthweight and type 2 diabetes risk is U-shaped, with a higher prevalence of type 2 diabetes observed in individuals with either low or high birthweight [20]. We therefore also aimed to explore this U-shaped association.

\section{Methods}

Participants The study methods have been described previously [19]. At the Hong Kong study centre, the HAPO study recruited 1667 pregnant women with a singleton pregnancy; all mothers underwent a 75 g OGTT at 24-32 weeks' gestation. Women of non-Chinese ancestry and women with glucose measurements beyond the setting of the HAPO study were excluded. Eligible participants were invited to attend a follow-up assessment between 2009 and 2013, when offspring were 7 years of age. The follow-up study of the children included a questionnaire, clinical examination and biochemical assessments. A total of 905 (58.2\%) mother-child pairs with term pregnancies ( $\geq 37$ weeks) and complete data on GWG were included for this analysis. The derivation of the eligible and analysis cohorts is shown in electronic supplementary material (ESM) Fig. 1.

This study was approved by the Clinical Research Ethics Committee of the Chinese University of Hong Kong. Research staff explained the study objective and procedures to both the mother and the child. The children's parents or legal guardians, as appropriate, provided written informed consent.

Exposure variables Maternal weight at delivery was abstracted by research staff from the medical records. Pre-pregnancy BMI was calculated as self-reported pre-pregnancy weight $(\mathrm{kg})$ divided by the square of the measured height $\left(\mathrm{m}^{2}\right)$. We defined total GWG as the difference between the mother's weight at delivery and her pre-pregnancy weight. Women were classified as gaining weight 'below', 'within', or 'exceeding' recommendations, according to their pre-pregnancy BMI category, on the basis of the 2009 IOM guidelines for healthy pregnancy weight gain [18].

Within each BMI category, the standardised GWG was computed as $\frac{\mathrm{GWG}_{i j}-\operatorname{mean}_{i}}{\mathrm{SD}_{i}}$ where: $\mathrm{GWG}_{i j}$ was the individual's GWG ( ${ }_{i}$ refers to the BMI categories and ${ }_{j}$ refers to each individual); $\operatorname{mean}_{i}$ was the mid-point of the range of GWG recommended by IOM in the $i$ th pre-pregnancy BMI category; $\mathrm{SD}_{i}$ was (upper ${ }_{i}-$ lower $\left._{i}\right) / 2$; and upper ${ }_{i}$ and lower $_{i}$ was the upper and lower ranges of GWG recommended by IOM in the $i$ th pre-pregnancy BMI category, respectively. For example, given that the recommended range of GWG in the 'underweight' group is from 12.5 to $18 \mathrm{~kg}, \operatorname{mean}_{i}=(18+12.5) /$ $2=15.25 \mathrm{~kg}$, and $\mathrm{SD}_{i}=(18-12.5) / 2=2.75 \mathrm{~kg}$.

Outcome variables Anthropometric indices including body weight, body height, waist and hip circumference, and biceps, triceps, subscapular and iliac skinfold thicknesses were measured. BMI was calculated as weight $/$ height $^{2}$. The sum of skinfold thickness was calculated as the sum of the biceps, triceps, subscapular and iliac skinfold thicknesses. Systolic and diastolic blood pressure (SBP and DBP) were measured in triplicate, and the mean readings were used. SBP and DBP for age-, sex-, and height-specific percentiles were computed based on data released from the National High Blood Pressure Education Program Working Group on High Blood Pressure in Children and Adolescents [21].

Participants were examined in the morning after an overnight fast. All individuals underwent an OGTT with a glucose load of $1.75 \mathrm{~g} / \mathrm{kg}$ body weight or a full $75 \mathrm{~g}$ glucose load if they weighed $\geq 42.8 \mathrm{~kg}$ (i.e. $1.75 \mathrm{~g} / \mathrm{kg} \times 42.8 \mathrm{~kg}$, which approximated to $75 \mathrm{~g}$ ), with blood taken at $0,15,30,60$ and $120 \mathrm{~min}$ to measure plasma glucose and insulin levels. Plasma glucose was measured by the hexokinase method, using an automated analyser (Hitachi 911; Boehringer Mannheim, Mannheim, Germany). Plasma insulin was analysed using 
an immunoassay analyser (Immulite 1000 Immunoassay System; Siemens, Munich, Germany). Fasting blood samples were also collected to measure lipid profile. AUCs for glucose $\left(\mathrm{AUC}_{\mathrm{glu}}\right)$ and insulin $\left(\mathrm{AUC}_{\mathrm{ins}}\right)$ during the OGTT at 0-120 min were calculated using the trapezoid rule.

Insulin sensitivity was determined using the HOMA-IR, calculated as (FPI $\times$ FPG $) \div 22.5$ [22], and the insulin sensitivity index (ISI), estimated as $10,000 \div$ square root of [FPI $\times$ $\mathrm{FPG} \times($ mean glucose during OGTT $) \times($ mean insulin during OGTT)] [23]. Beta cell function was assessed using HOMA- $\beta$, calculated as FPI $\times 20 \div(\mathrm{FPG}-3.5)$ [22]. The insulinogenic index (IGI) was calculated as (insulin during OGTT at $30 \mathrm{~min}-0 \mathrm{~min}$ ) $\div$ (glucose during OGTT at $30 \mathrm{~min}$ $-0 \mathrm{~min}$ ) [24], and pancreatic beta cell function was calculated as $\mathrm{AUC}_{\text {ins }} \div \mathrm{AUC}_{\text {glu }}[25]$.

Statistical analysis All analyses were performed using either SPSS for Windows v22 (SPSS, IBM, Chicago, IL, USA) or R 2.15.1 (www.r-project.org/). $p<0.05$ was considered statistically significant. Data are presented as percentage $(n)$, mean $\pm \mathrm{SD}$, or median $(\mathrm{Q} 1-\mathrm{Q} 3)$. Comparison between IOM categories of GWG was performed by $\chi^{2}$ test, one-way ANOVA or Kruskal-Wallis test, as appropriate.

Each normally distributed trait was winsorised by replacing any data value above the mean plus $4 \mathrm{SD}$ of the sample data by the mean plus $4 \mathrm{SD}$, and any value below the mean minus 4 SD by the mean minus $4 \mathrm{SD}$. A total of $0.5 \%$ of the data were replaced. All traits were then adjusted for covariates using linear regression as follows: model 1-child's sex, age and/ or height as appropriate; model 2-model $1+$ family history (maternal pre-pregnant BMI and/or maternal current hypertensive status and/or maternal and paternal current diabetes status, as appropriate); model 3-model $2+$ environmental factors during prenatal (parity, maternal age, $\mathrm{AUC}_{\mathrm{glu}}$ during pregnancy, mode of delivery [spontaneous, low forceps/vacuum, mid-forceps/vacuum, assisted/spontaneous breech, total breech, internal version/breech, primary Caesarean section or repeat Caesarean section] and gestational age at delivery) and postnatal periods (history of breastfeeding and childhood exercise level); model 4 -model $3+$ birthweight; and model 5 - model $4+$ childhood BMI. Individuals with missing data points for any variables included in the linear regression model were removed from the analysis. A mean of $3.9 \%$ of individuals were excluded in each regression analysis. The resulting residuals were transformed to a $z$ score for normally distributed traits, and transformed to approximate normality using an inverse standard normal function for the skewed traits.

In the primary analysis, associations between IOM recommendations for GWG and transformed cardiometabolic risk factors during childhood were tested using a linear regression model. Two dummy variables were used to code for the GWG categories, 'GWG below IOM recommendation' and 'GWG exceeding IOM recommendation'. Each group was compared with the reference group 'GWG within IOM recommendation'. To investigate for non-linear associations, both linear and quadratic terms of standardised GWG were included in the regression model. The significant $p$ values obtained from the regression analyses for various cardiometabolic traits are presented as a range, between the lowest and highest values among all traits. The following terminology is used for $p$ values: $p_{\text {below }}$, the $p$ value for the comparison of GWG below the IOM recommendations with GWG within the IOM recommendations; $p_{\text {exceeding }}$, the $p$ value for the comparison of GWG exceeding the IOM recommendations with GWG within the IOM recommendations; $p_{\text {linear }}$ and $p_{\text {quadratic }}$, the $p$ values of the linear and quadratic terms respectively, obtained from linear regression analyses.

\section{Results}

Cohort description The characteristics of the women and newborn offspring included in this analysis are listed in ESM Table 1. Among the 905 mothers, the mean pre-pregnancy BMI was $20.9 \pm 2.9 \mathrm{~kg} / \mathrm{m}^{2}$, and the prevalence of women who were overweight or obese was $8.3 \%$. The mean weight change from pre-pregnancy to delivery was $15.2 \pm 4.36 \mathrm{~kg}$, with $17.2 \%$ having gained weight below, $41.8 \%$ weight within and $41.0 \%$ weight exceeding the IOM recommendations. The proportions of women who gained insufficient, adequate and excessive weight differed between pre-pregnancy BMI categories $\left(p=1.4 \times 10^{-6}\right)$. In general, women who gained excessive weight during pregnancy were younger and had longer gestational periods, a higher primary Caesarean delivery rate and a greater BMI before pregnancy, at delivery and at 7 years after delivery. Offspring born to mothers with excessive GWG were heavier and longer, showed greater adiposity and had higher umbilical cord blood C-peptide concentrations at birth.

IOM categories of GWG and childhood cardiometabolic risk With adjustments for children's sex, age and/or height, the offspring of mothers who gained excessive weight during pregnancy had a larger body size (greater height and weight), greater BMI, waist circumference and hip circumference, and higher blood pressure (higher DBP, SBP and DBP percentiles), FPI, $2 \mathrm{~h}$ insulin and $\mathrm{AUC}_{\text {ins }}$, were more insulin resistant (higher HOMA-IR and lower Matsuda ISI) and showed a greater insulin response (higher HOMA- $\beta$ and IGI, and increased pancreatic beta cell function) than the offspring of mothers who achieved the weight gain target during pregnancy $\left(4.6 \times 10^{-9}<p<0.0390\right.$; model 1 in Table 1 ; see also ESM Table 2 [model 1] and Fig. 1). Moreover, women with GWG below the IOM recommendations had children with higher DBP percentiles, $\mathrm{AUC}_{\mathrm{ins}}$ and pancreatic beta cell function, and a lower Matsuda ISI, than women whose GWG was 


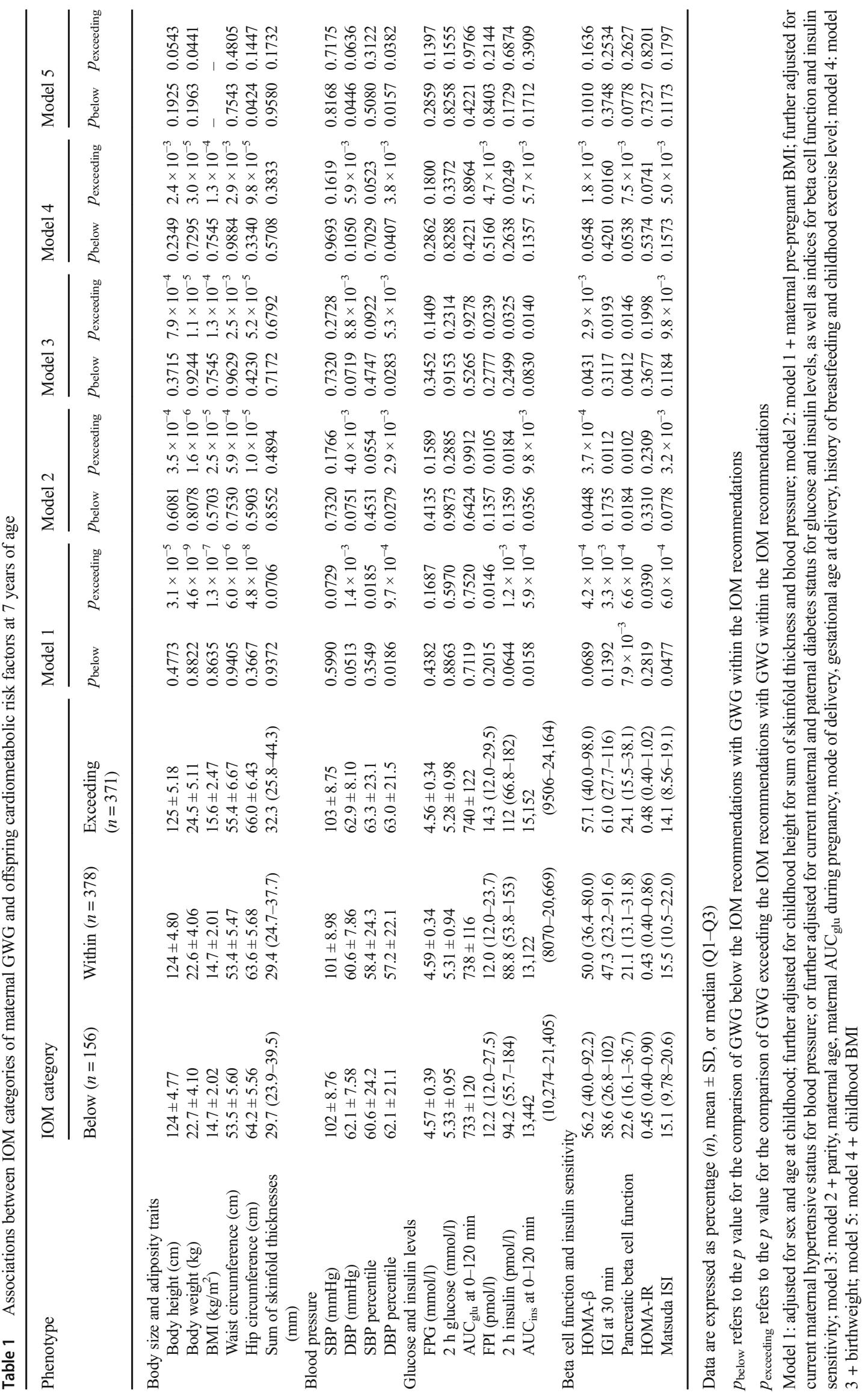


a

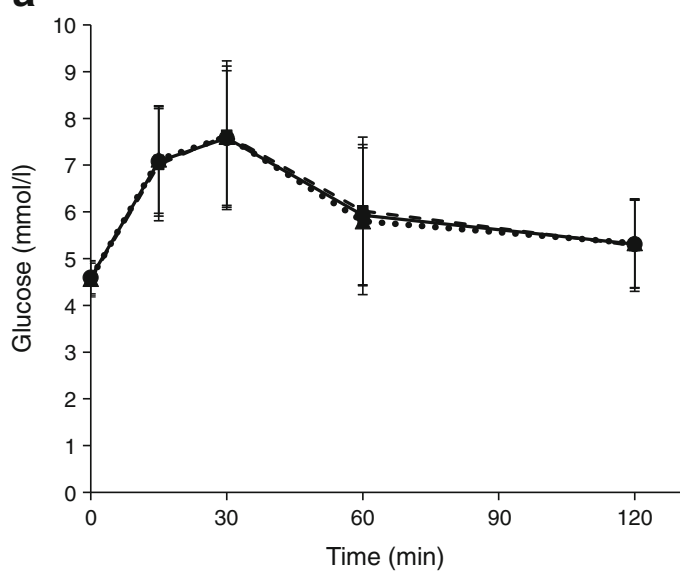

b

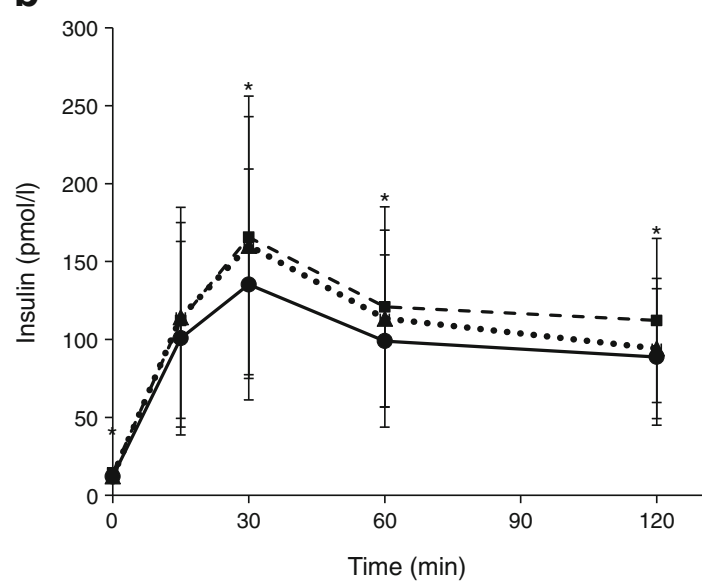

Fig. 1 (a) Plasma glucose and (b) insulin concentrations at 0, 15, 30, 60 and 120 min during the OGTTs, stratified by IOM recommendations of GWG categories. Data are presented as mean $\pm \mathrm{SD}$ and median \pm absolute median deviation for glucose and insulin concentration, respectively, according to the time intervals. Black triangles with dotted line, group below recommendations; black circles with solid line, group within recommendations; black squares with dashed line, group exceeding recommendations. $* p<0.05$ for the association across the IOM categories at each time point, with adjustments for children's sex and age (ANOVA)

within the IOM recommendations $\left(7.9 \times 10^{-3}<p<0.0477\right)$ (model 1 in Table 1; see also model 1 in ESM Table 2). Most of these associations were independent of other covariates, including maternal pre-pregnancy BMI, family histories of hypertension and diabetes in model 2, perinatal and childhood environmental factors in model 3, as well as birthweight in model 4 (Table 1). We still found significant associations between excessive GWG and body size (height and weight), adiposity traits (BMI, waist circumference and hip circumference), blood pressure (DBP and DBP percentiles), insulin levels (FPI, $2 \mathrm{~h}$ insulin and $\mathrm{AUC}_{\mathrm{ins}}$ ), insulin sensitivity (Matsuda ISI) and beta cell function (HOMA- $\beta$, IGI and pancreatic beta cell function) ( $p_{\text {exceeding }}<0.05$ ), although the association of GWG with HOMA-IR and SBP percentile was no longer significant in models $2-4\left(p_{\text {exceeding }}>0.05\right)$
(Table 1). On the other hand, the association of inadequate GWG with DBP percentile $\left(0.0279<p_{\text {below }}<0.0407\right)$ and pancreatic beta cell function $\left(0.0184<p_{\text {below }}<0.0538\right)$ was marginally significant, while its association with $\mathrm{AUC}_{\text {ins }}(p$ below $=0.0158,0.0356,0.0830$ and 0.1357 in models $1,2,3$ and 4 , respectively) and Matsuda ISI ( $p_{\text {below }}=0.0477,0.0778$, 0.1184 and 0.1573 in models $1,2,3$ and 4 , respectively) were slightly mitigated in models $2-4$ (Table 1 ). However, the observed associations for both excessive and inadequate GWG in models 1-4 were notably attenuated when conditional on childhood BMI (model 5 in Table 1).

Standardised GWG and childhood cardiometabolic risk To explore the non-linear/quadratic relationship between maternal GWG and childhood cardiometabolic traits, analyses were repeated using the standardised GWG. In model 1 with adjustments for children's sex, age and/or height, there was a Ushaped relationship between standardised GWG and adverse cardiometabolic risk profile in the offspring, with higher weight, hip circumference, blood pressure (DBP and DBP percentile) and $\mathrm{AUC}_{\mathrm{ins}}$, increased risks of insulin resistance (Matsuda ISI) and an enhanced insulin response (pancreatic beta cell function) in the children of women with either more or less weight gain than recommended during pregnancy $\left(1.4 \times 10^{-3}<p_{\text {quadratic }}<0.0282\right)$ (model 1 in Table 2; see also model 1 in ESM Table 3 and ESM Fig. 2). Further adjustments for maternal pre-pregnancy BMI, family histories of hypertension and diabetes, perinatal and childhood environmental factors, and children's birthweight in models 2-4 attenuated, but did not eliminate, the quadratic associations for DBP ( $p$ quadratic $=0.0109,0.0422,0.0720$ and 0.0808 in models 1,2 , 3 and 4 , respectively), DBP percentile $\left(p_{\text {quadratic }}=6.4 \times 10^{-3}\right.$, 0.0254, 0.0417 and 0.0477 in models $1,2,3$ and 4, respectively), $\mathrm{AUC}_{\text {ins }}\left(p_{\text {quadratic }}=1.4 \times 10^{-3}, 0.0173,0.0312\right.$ and 0.0381 in models $1,2,3$ and 4 , respectively), pancreatic beta cell function $\left(p_{\text {quadratic }}=3.7 \times 10^{-3}, 0.0302,0.0455\right.$ and 0.0469 in models 1, 2, 3 and 4, respectively) and Matsuda ISI $\left(p_{\text {quadratic }}=4.9 \times 10^{-3}, 0.0401,0.0462\right.$ and 0.0454 in models 1, 2, 3 and 4, respectively). In addition, we observed a positive linear relationship for weight $\left(7.5 \times 10^{-4}<p_{\text {linear }}<\right.$ 0.0224 and $\left.p_{\text {quadratic }}>0.05\right)$ and hip circumference $(5.8 \times$ $10^{-3}<p_{\text {linear }}<0.0573$ and $p_{\text {quadratic }}>0.05$ ) in models $2-4$ (Table 2). Only the association between standardised GWG and DBP percentile remained significant after adjusting for childhood BMI (model 5 in Table 2).

\section{Discussion}

Accumulating evidence suggests that adiposity and related cardiometabolic risk factors in the offspring are influenced by maternal weight gain during pregnancy. Using the follow-up data of 905 mother-child pairs from the HAPO 


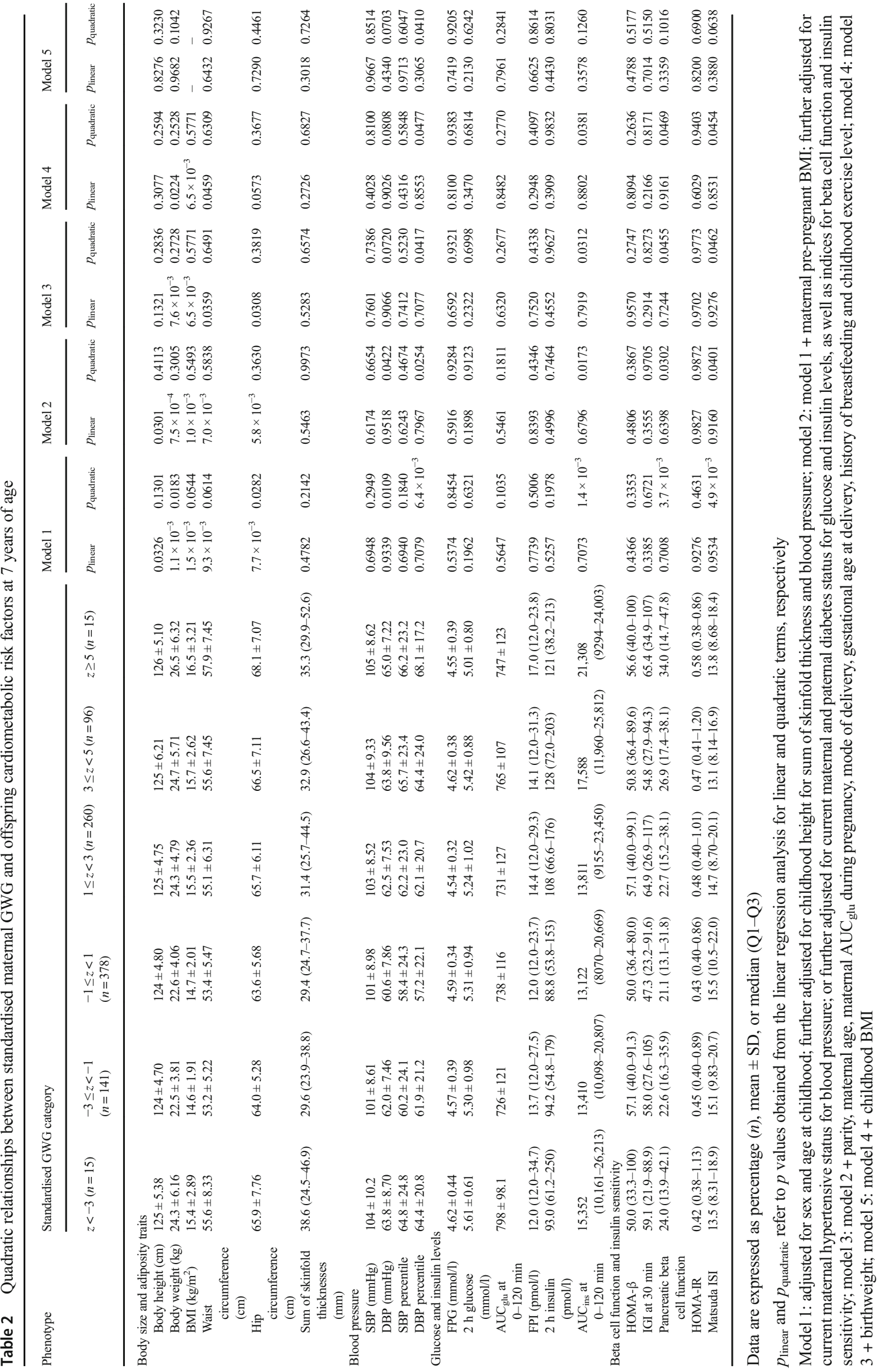


study, we extended observations from earlier studies by (1) examining both linear and non-linear associations between maternal GWG and cardiometabolic risk factors in offspring aged 7 years, and (2) including data from OGTTs. To achieve a better understanding of the causal pathways, we have also systematically considered the potential confounding effects of the clinical risk factors in these relationships.

Consistent with the majority of previous studies conducted in infancy [8], early childhood [7, 11, 13] and adulthood [15-17], we confirm that maternal GWG was positively associated with body size and adiposity in offspring aged 7 years. These associations were also found to be independent of maternal pre-pregnancy BMI, shared familial genetics and environment, and environmental factors during the prenatal, perinatal and postnatal periods. Two recent studies investigated the association between pregnancy weight gain and childhood body composition assessed by dual x-ray absorptiometry (DXA) but the available evidence is inconclusive $[9,26]$. Skinfold thickness, which is widely measured in children, has been suggested as a simple means of estimating body composition. In Project Viva, which included 1044 mother-child pairs, Oken et al reported an increase in the sum of skinfold thickness in the offspring at 3 years of age, along with an increase in maternal GWG [13]. However, we did not observe such association in the present study $\left(p_{\text {exceeding }}=0.0706\right)($ model 1 in Table 1$)$.

Several studies revealed that in utero exposure to excessive GWG predicts obesity-related cardiometabolic risk factors in both childhood [11, 13] and adulthood [15-17]. However, the existing body of literature is small and inconsistent. There are few data exploring the links between maternal GWG and insulin sensitivity and beta cell function in the offspring. In this context, we have obtained novel insights through examining the associations for insulin action and response indices calculated using OGTT data at the follow-up visit. One of the most important findings from this study was that, independently of maternal pre-pregnancy obesity and glucose level during pregnancy, maternal GWG had a U-shaped relationship with increased odds of childhood insulin resistance and hypertension, with higher DBP levels, greater $\mathrm{AUC}_{\mathrm{ins}}$ and pancreatic beta cell function, and a lower Matsuda ISI in the children whose mothers gained more or less weight than recommended during pregnancy. The observed association for increased pancreatic beta cell function and reduced insulin sensitivity may reflect an initial compensation by beta cells for the obesity-induced insulin resistance, by increasing insulin secretion [27]. Similar to previous reports [11, 15-17], adjustment for childhood BMI appreciably alters these relationships.

In support of our findings, several research groups have illustrated a positive association between weight gain during pregnancy and both SBP and DBP in children $[10,11]$ and adults $[16,17]$, whereas others did not make similar observations $[14,15]$. Two studies further examined the impact of maternal GWG on insulin resistance in the offspring assessed by HOMA-IR and found no evidence of association [12, 17], although Hrolfsdottir et al observed a significant association in male offspring [17]. Interestingly, none of the studies reported a U-shaped relationship between GWG and obesity-related cardiometabolic risk factors [11-13, 15-17]. The discordant findings may have arisen from the method used to calculate the total weight gain during pregnancy. As it was not always possible to obtain information on maternal weight just before conception or at delivery, the duration of the specified period for assessing GWG varies among studies. Using dynamic 5-point measurements of glucose and insulin levels during the OGTTs in this study rather than using basal measurement alone should provide a more comprehensive assessment of beta cell function and insulin sensitivity. Furthermore, the different ages of offspring participants between studies may also account for variation in findings. Larger studies are warranted to confirm the Ushaped relationship.

One potential mechanism accounting for our observed associations between GWG and cardiometabolic outcomes in the offspring is the role of shared familial genes and behaviours (e.g. diet, physical activity and socioeconomic status); for example, offspring may inherit their mother's genetic potential to gain weight. Moreover, mothers and children may share obesogenic dietary patterns (e.g. a preference for fatty food) and lifestyle habits (e.g. lower levels of physical activity), particularly in early childhood [28], which may link greater maternal GWG with unfavourable outcomes in the offspring in later life. To take into account the factor of shared genes and behaviours, we have attempted to statistically adjust for maternal pre-pregnancy BMI, family histories of hypertension and diabetes, and characteristics reflecting the early and childhood environments. Most of the associations between GWG and childhood cardiometabolic risk were slightly attenuated but remained statistically significant, suggesting that these associations were not mainly driven by shared familial characteristics in our study. These findings point towards an alternative mechanism, implicating the potential effect of intrauterine environment.

There is increasing recognition of the potential metabolic impact of maternal adiposity, suggesting that high maternal plasma concentrations of glucose, NEFA and amino acids result in a sustained modulation of appetite control, neuroendocrine functioning or energy metabolism in the developing fetus. The fetus may thus become more vulnerable to an obesogenic environment, leading to adiposity and related cardiometabolic diseases in later life. Human studies of individuals undergoing bariatric surgery highlighted the impact of intrauterine environment on health outcomes in the 
offspring. A study that included 49 mothers and their 111 children aged 2.5-26 years demonstrated that offspring born after maternal biliopancreatic diversion bariatric surgery had a markedly lower prevalence of macrosomia, a reduced risk of severe obesity, greater insulin sensitivity and improved lipid profiles in adolescence, compared with their siblings born prior to the surgery [29]. In addition, Shankar et al developed a model of maternal obesity in rats based on overfeeding that allowed for a comparison in genetically identical individuals of the association between exposure to maternal obesity in utero and risk of obesity in the offspring. Shankar et al's findings confirmed that maternal obesity at conception programmes lifelong obesity in the offspring [30]. Notably, our findings suggest that the effect of maternal GWG on childhood cardiometabolic risk is not confined to the upper and lower extremes of GWG, but rather this relationship is a U-shaped continuum. Contrary to the tenets of Barker's hypothesis, which focuses on extreme prenatal challenges as being crucial to the disease pathway, a new global landscape of disease is emerging in which the relationship between developmental environment and disease risk is a U-shaped continuum within the physiological range [31]. It also suggests that subtle variations in prenatal experience (e.g. maternal nutrition) might affect the risk of disease, and this effect can be amplified at all levels by a plentiful environment (e.g. unhealthy diet and lifestyle) in adulthood [31]. The lowest risk of disease occurs when both developmental and adult environments are optimal.

The current study has unique strengths. Our mother-child cohort was prospectively designed, with comprehensive clinical assessments of childhood cardiometabolic risk factors including glucose and insulin levels during OGTTs. The availability of detailed measures collected in early life and childhood permitted meaningful and stepwise adjustment for a large number of potential confounders. Owing to the design of the HAPO study, the mothers and their doctors were blinded to the OGTT results and hence did not receive any dietary or medical treatment for hyperglycaemia during pregnancy, which would have confounded similar analyses in other studies.

There are, however, several limitations of the study. First, pre-pregnancy weight was self-reported by the mothers, and thus the estimation of GWG may be inaccurate. We noted that there was a strong correlation between the self-reported prepregnancy weight and the weights measured at the pregnancy OGTT visits $\left(r^{2}=0.899\right)$, supporting the validity of the selfreported data. Second, the IOM recommendations for GWG were developed in a cohort consisting largely of healthy white women, and used the standard BMI thresholds to define the categories of GWG. As adults in Asia have different BMI classification thresholds for overweight and obesity, further studies with sufficiently large sample sizes are required to determine the optimal GWG for Asian populations. Third, $41.8 \%(n=697)$ of participants were non-eligible, declined the follow-up visit or were not contactable. Compared with children who attended the follow-up visit, we found no evidence of discrepancy in the distributions of GWG between two groups (ESM Table 4). Therefore, the observed associations are unlikely to be due to selection bias. Fourth, our findings were restricted to individuals of Chinese ancestry and children aged 6-8 years, with limited generalisability. Last, we were not able to explore the associations using GWG at different stages of pregnancy because repeated measures of maternal weight were not recorded in this study.

In conclusion, we found evidence of linkage between GWG and several cardiometabolic risk factors in the offspring aged 7 years, independent of maternal BMI prior to pregnancy and glucose levels during pregnancy. These findings have important implications for prevention and treatment. Pregnancy may be a potential window of opportunity for intervention through modifiable behaviours, including maternal nutrition and physical activity. Although limiting excessive GWG may help minimise the intergenerational cycle of obesity, the benefits of lower weight gain must be balanced against other cardiometabolic risks (e.g. hypertension and insulin resistance) and risk of stunted growth in the offspring if maternal GWG is inadequate. Finally, long-term follow-up of these children is necessary to evaluate the effect of maternal GWG on cardiometabolic risk in adolescence and adulthood.

Acknowledgements We thank the HAPO study steering committee for initiating and conducting the original study, and for their kind help and support. We would especially like to thank A. R. Dyer (Feinberg School of Medicine, Northwestern University, Chicago, IL, USA) for reviewing the manuscript and providing supportive comments. We are also grateful to all the study participants for their contribution. Special thanks are extended to all nursing and medical staff at the PWH Diabetes and Endocrine Centre for their dedication and professionalism.

Data availability The data supporting the findings of this study are available on request from the corresponding authors (WHT and RCWM). The data are not publicly available as they contain information that could compromise the privacy or consent of the research participants.

Funding The HAPO study was funded by the National Institute of Child Health and Human Development (grant no. R01-HD34242) and the National Institute of Diabetes and Digestive and Kidney Diseases (grant no. R01-HD34243). The HAPO follow-up study at the Hong Kong Center was supported by the funding from the General Research Fund of the Research Grants Council of the Hong Kong SAR, China (grants CUHK 473408 and, in part, CUHK 471713).

Duality of interest The authors declare that there is no duality of interest associated with this manuscript.

Contribution statement WHT and RCWM are the principal investigators of the HAPO follow-up study; they both conceived and coordinated the investigation. LYY, CHTT and WHT were responsible for data preparation and statistical analyses. AML, XY and JCNC contributed 
significantly to the conception and design of the study. RO contributed to data acquisition and monitoring of field work and patient safety throughout the study. CHTT, WHT and RCWM wrote the first draft of the manuscript. YH, MHMC and CSH performed the biochemical and insulin measurements from blood samples. All authors undertook revisions and contributed intellectually to the development of the report. All authors listed made significant contributions to the study and gave final approval of the version to be published. WHT and RCWM are the guarantors of this work; they had full access to all the data in the study and take responsibility for the decision to submit for publication.

Open Access This article is distributed under the terms of the Creative Commons Attribution 4.0 International License (http:// creativecommons.org/licenses/by/4.0/), which permits unrestricted use, distribution, and reproduction in any medium, provided you give appropriate credit to the original author(s) and the source, provide a link to the Creative Commons license, and indicate if changes were made.

\section{References}

1. Karter AJ, Rowell SE, Ackerson LM et al (1999) Excess maternal transmission of type 2 diabetes. The Northern California Kaiser Permanente Diabetes Registry. Diabetes Care 22:938-943

2. Tam CH, Wang Y, Luan J et al (2014) Maternal history of diabetes is associated with increased cardiometabolic risk in Chinese. Nutr Diabetes 4:e112

3. Ravelli AC, van der Meulen JH, Michels RP et al (1998) Glucose tolerance in adults after prenatal exposure to famine. Lancet 351: 173-177

4. Wadhwa PD, Buss C, Entringer S, Swanson JM (2009) Developmental origins of health and disease: brief history of the approach and current focus on epigenetic mechanisms. Semin Reprod Med 27:358-368

5. Pettitt DJ, Nelson RG, Saad MF, Bennett PH, Knowler WC (1993) Diabetes and obesity in the offspring of Pima Indian women with diabetes during pregnancy. Diabetes Care 16:310-314

6. Reynolds CM, Gray C, Li M, Segovia SA, Vickers MH (2015) Early life nutrition and energy balance disorders in offspring in later life. Nutrients 7:8090-8111

7. Wrotniak BH, Shults J, Butts S, Stettler N (2008) Gestational weight gain and risk of overweight in the offspring at age $7 \mathrm{y}$ in a multicenter, multiethnic cohort study. Am J Clin Nutr 87:18181824

8. Badon SE, Dyer AR, Josefson JL (2014) Gestational weight gain and neonatal adiposity in the hyperglycemia and adverse pregnancy outcome study-North American region. Obesity 22:1731-1738

9. Crozier SR, Inskip HM, Godfrey KM et al (2010) Weight gain in pregnancy and childhood body composition: findings from the Southampton Women's Survey. Am J Clin Nutr 91:1745-1751

10. Oken E, Rifas-Shiman SL, Field AE, Frazier AL, Gillman MW (2008) Maternal gestational weight gain and offspring weight in adolescence. Obstet Gynecol 112:999-1006

11. Fraser A, Tilling K, Macdonald-Wallis C et al (2010) Association of maternal weight gain in pregnancy with offspring obesity and metabolic and vascular traits in childhood. Circulation 121:2557-2564

12. Maftei O, Whitrow MJ, Davies MJ, Giles LC, Owens JA, Moore VM (2015) Maternal body size prior to pregnancy, gestational diabetes and weight gain: associations with insulin resistance in children at 9-10 years. Diabet Med 32:174-180

13. Oken E, Taveras EM, Kleinman KP, Rich-Edwards JW, Gillman MW (2007) Gestational weight gain and child adiposity at age 3 years. Am J Obstet Gynecol 196:322.e1-322.e8
14. Laor A, Stevenson DK, Shemer J, Gale R, Seidman DS (1997) Size at birth, maternal nutritional status in pregnancy, and blood pressure at age 17: population based analysis. BMJ 315:449-453

15. Mamun AA, O'Callaghan M, Callaway L, Williams G, Najman J, Lawlor DA (2009) Associations of gestational weight gain with offspring body mass index and blood pressure at 21 years of age: evidence from a birth cohort study. Circulation 119:1720-1727

16. Hochner H, Friedlander Y, Calderon-Margalit R et al (2012) Associations of maternal prepregnancy body mass index and gestational weight gain with adult offspring cardiometabolic risk factors: the Jerusalem Perinatal Family Follow-up Study. Circulation 125:1381-1389

17. Hrolfsdottir L, Rytter D, Olsen SF et al (2015) Gestational weight gain in normal weight women and offspring cardio-metabolic risk factors at 20 years of age. Int J Obes 39:671-676

18. Institute of Medicine (US) and National Research Council (US) Committee to Reexamine IOM Pregnancy Weight Guidelines (2009) In: Rasmussen KM, Yaktine AL (eds) Weight gain during pregnancy: reexamining the guidelines. The National Academies Press, Washington, DC

19. Tam WH, Ma RCW, Ozaki R et al (2017) In utero exposure to maternal hyperglycemia increases childhood cardiometabolic risk in offspring. Diabetes Care 40:679-686

20. McCance DR, Pettitt DJ, Hanson RL, Jacobsson LT, Knowler WC, Bennett PH (1994) Birth weight and non-insulin dependent diabetes: thrifty genotype, thrifty phenotype, or surviving small baby genotype? BMJ 308:942-945

21. National High Blood Pressure Education Program Working Group on High Blood Pressure in Children and Adolescents (2004) The fourth report on the diagnosis, evaluation, and treatment of high blood pressure in children and adolescents. Pediatrics 114:555-576

22. Matthews DR, Hosker JP, Rudenski AS, Naylor BA, Treacher DF, Turner RC (1985) Homeostasis model assessment: insulin resistance and beta-cell function from fasting plasma glucose and insulin concentrations in man. Diabetologia 28:412-419

23. Matsuda M, DeFronzo RA (1999) Insulin sensitivity indices obtained from oral glucose tolerance testing: comparison with the euglycemic insulin clamp. Diabetes Care 22:1462-1470

24. Phillips DI, Clark PM, Hales CN, Osmond C (1994) Understanding oral glucose tolerance: comparison of glucose or insulin measurements during the oral glucose tolerance test with specific measurements of insulin resistance and insulin secretion. Diabet Med 11: 286-292

25. Stumvoll M, Mitrakou A, Pimenta W et al (2000) Use of the oral glucose tolerance test to assess insulin release and insulin sensitivity. Diabetes Care 23:295-301

26. Gale CR, Javaid MK, Robinson SM, Law CM, Godfrey KM, Cooper C (2007) Maternal size in pregnancy and body composition in children. J Clin Endocrinol Metab 92:3904-3911

27. Kasuga M (2006) Insulin resistance and pancreatic beta cell failure. J Clin Invest 116:1756-1760

28. Birch LL (1999) Development of food preferences. Annu Rev Nutr 19:41-62

29. Smith J, Cianflone K, Biron S et al (2009) Effects of maternal surgical weight loss in mothers on intergenerational transmission of obesity. J Clin Endocrinol Metab 94:4275-4283

30. Shankar K, Harrell A, Liu X, Gilchrist JM, Ronis MJ, Badger TM (2008) Maternal obesity at conception programs obesity in the offspring. Am J Physiol Regul Integr Comp Physiol 294:R528-R538

31. Godfrey KM, Gluckman PD, Hanson MA (2010) Developmental origins of metabolic disease: life course and intergenerational perspectives. Trends Endocrinol Metab 21:199-205 